\title{
LA ALIMENTACIÓN ESCOLAR EN LAS INSTITUCIONES EDUCATIVAS PÚBLICAS DE COLOMBIA. ANÁLISIS NORMATIVO Y DE LA POLÍTICA PÚBLICA ALIMENTARIA*
}

\author{
Germán Alfonso López Daza** \\ Katherin Torres $P^{* * *}$ \\ Carlos Fernando Gómez García * *
}

Fecha de recepción: 2 de febrero de 2017

Fecha de evaluación: 27 de abril de 2017

Fecha de aprobación: 16 de mayo de 2017

Artículo de investigación

DOI: http://dx.doi.org/10.18359/prole.3043

Forma de citación: López, G. A., Torres, K. \& Gómez, C. F. (2017). La alimentación escolar en las instituciones educativas públicas de Colombia. Análisis normativo y de la política pública alimentaria. Revista Prolegómenos Derechos y Valores, 20, 40, 97-112. DOI: http://dx.doi.org/10.18359/prole.3043

\section{Resumen}

Este artículo presenta los resultados de la investigación "La alimentación escolar en las instituciones educativas públicas de Colombia", desarrollada por el grupo de investigación Nuevas Visiones del Derecho de la Universidad Surcolombiana y financiada por la Organización de las Naciones Unidas para la Alimentación y la Agricultura y el Observatorio del Derecho a la Alimentación en América Latina y el Caribe. El objetivo principal consistió en determinar si los productos alimenticios que se venden en las tiendas escolares de las instituciones educativas públicas de Colombia, cumplen con los estándares y obligaciones prescritas en la ley 1355/2009 que adoptó medidas de prevención y atención frente a la obesidad y las enfermedades crónicas no transmisibles. Esta investigación tuvo un enfoque mixto, ya que con la ayuda de los datos estadísticos que arrojaron las respuestas de los derechos de petición, se hizo un análisis cualitativo del contenido de las políticas públicas de los departamentos, municipios y colegios.

- Artículo resultado de la investigación desarrollada por el Grupo de Investigación Nuevas Visiones del Derecho de la Universidad Surcolombiana y financiada por la Organización de las Naciones Unidas para la Alimentación y la Agricultura y el Observatorio del Derecho a la Alimentación en América Latina y el Caribe.

* Ph.D. Droit Constitutionnel, Université de Paris II (París, Francia). Coordinador de la maestría en Derecho Público, profesor e investigador del grupo Nuevas Visiones del Derecho de la Universidad Surcolombiana (Neiva, Colombia). Correo electrónico: germanlo@usco.edu.co

Profesora e investigadora del grupo Nuevas Visiones del Derecho de la Universidad Surcolombiana (Neiva, Colombia). Abogada de la Universidad Surcolombiana y magíster en Derecho con énfasis en Responsabilidad Contractual y Extracontractual Civil y del Estado de la Universidad Externado de Colombia (Bogotá, Colombia). Correo electrónico: katherin. torres@usco.edu.co

Profesor e investigador del grupo Nuevas Visiones del Derecho de la Universidad Surcolombiana (Neiva, Colombia). Abogado de la Universidad Surcolombiana y candidato a magíster en Derecho Público de la Universidad Santo Tomás (Bogotá, Colombia). Correo electrónico: carlos.gomez@usco.edu.co 


\title{
Palabras clave:
}

Seguridad alimentaria, alimentación escolar, política pública alimentaria.

\section{SCHOOL NUTRITION IN THE COLOMBIAN PUBLIC EDUCATIONAL INSTITUTIONS. ANALYSIS OF THE REGULATION AND THE PUBLIC NUTRITION POLICY}

\begin{abstract}
Summary
This article presents the results of the research titled "School Nutrition in the Colombian Public Institutions". It was developed by the research group New Tendencies of Law from the Surcolombiana University, and funded by the Right to Food and Nutrition Watch of the FAO. The main goal consisted in determining whether the food products sold in the shops of the Colombian public institutions, achieved the standards and obligations prescribed by the Law 1355 of 2009. This law adopted preventive and attentive measures against obesity and chronic non-communicable diseases. This research had a mixed approach thanks to the help provided by the statistical data retrieved from the written petition's responses which made possible for a qualitative analysis of the public policy in departments, townships and schools.
\end{abstract}

\section{Keywords:}

Food security, school nutrition, public food policy

\section{A ALIMENTAC̄̃O ESCOLAR NAS INSTITUICÕ̃ES DE ENSINO PÚBLICAS DA COLÔMBIA. ANÁLISE NORMATIVA E DA POLIÍTICA PÚBLICA ALIMENTARIA}

\section{Resumo}

Este artigo apresenta os resultados da pesquisa intitulada "A alimentação escolar nas instituições de ensino públicas na Colômbia", desenvolvida pelo grupo de pesquisa Novas Visões do Direito da Universidade Surcolombiana e a qual foi financiada pelo Observatório do Direito à Alimentação da FAO. O principal objetivo foi determinar se os produtos alimentares vendidos nas lojas escolares das instituições de ensino públicas da Colômbia, cumprem com os padrões e obrigações prescritas na Lei 1355 de 2009, que adotou medidas de prevenção e tratamento contra a obesidade e as doenças crônicas não transmissíveis. Esta pesquisa teve uma abordagem mista, já que com a ajuda dos dados estatísticos que lançaram as respostas dos direitos de petição, se fez uma análise qualitativa do conteúdo das políticas públicas dos departamentos, municípios e colégio.

\section{Palavras-chave:}

Segurança alimentar, alimentação escolar, política pública alimentar.

\section{Introducción}

El proyecto de investigación que dio origen a este artículo lo financió la Organización de las Naciones Unidas para la Alimentación y la
Agricultura y el Observatorio del Derecho a la Alimentación en América Latina y el Caribe. Los resultados se presentaron en el VI Encuentro Regional del Observatorio del Derecho a la Alimentación en América Latina y el Caribe 
realizado en Montevideo (Uruguay) entre el 30 de noviembre y el 2 de diciembre del 2016.

La obesidad, desde el punto de vista teórico se "define como un incremento del peso corporal, a expensas preferentemente del aumento del tejido adiposo" (Colomer, 2005, p. 79); y a pesar de que tal vez la obesidad tuviera otras percepciones en la sociedad, como "estatus de belleza", en la actualidad se cataloga como una enfermedad crónica no transmisible, que para la población infantil genera graves consecuencias, puesto que los niños y adolescentes:

[...] presentan bajo rendimiento escolar, problemas de aprendizaje, ausentismo, así como riesgos en su salud y bienestar en etapas posteriores de la vida. Para la sociedad implica no solo el alto costo de la atención en salud, sino una disminución en la productividad y la calidad de vida de la población (Fajardo, 2012, p. 6).

La obesidad infantil ha sido materia de estudio en múltiples investigaciones en Colombia ${ }^{1}$. Igualmente, en estudios internacionales se ha determinado su origen en varias causas como las hereditarias. Por ejemplo, se encuentra en algunas investigaciones médicas, como la de Stunkard et al. (1986, citados en Chueca, Azcona y Oyarzábal, 2002), una conexión importante entre los índices de masa corporal en niños adoptados y sus padres biológicos, lo que sustentaría las causas genéticas. También se han determinado causas ambientales, que califican algunos autores (Muzo, 2003) como las que con mayor periodicidad condicionan el cúmulo anormal de peso; y falta de ejercicio físico, causado por una serie de elementos que Baile (2007) los precisa en el transporte, el entorno urbanístico poco favorable al ejercicio físico y el sedentarismo (televisión, videojuegos, redes sociales, etc.).

Ver entre otros los estudios médicos de Eulalia María Amador Rodero y Leslie Piedad Montealegre (2011) y de Esperanza Fajardo Bonilla y Luis Alberto Ángel Arango (2012).
El problema de la obesidad infantil es un asunto que en Colombia crece exponencialmente. Según datos estadísticos, el 5,2 por ciento de los menores de cinco años tiene sobrepeso de acuerdo con la Encuesta Nacional de la Situación Nutricional (Ensin) 2010 y el documento del 2012, Nutrición en Colombia II. Actualización del estado nutricional con implicaciones de política, liderado por el Banco Interamericano de Desarrollo, en donde se dice que:

La mayor prevalencia se observa en niños cuyas madres tienen educación superior $(6,2 \%)$ y pertenecen a un SISBEN igual o mayor a $4(6,3 \%)$ y la más baja en hijos de mujeres sin educación $(2,3 \%)$ y pertenecientes al SISBEN 1 (4,3\%). Si se compara la prevalencia entre diferentes comunidades étnicas, interesantemente la mayor prevalencia se observa en la población indígena $(6,8 \%)$, seguida por la población clasificada como otro (5,3\%) y afro-descendiente $(4,0 \%)$ (BID, 2012, p. 11).

Desde de esta perspectiva, el entorno infantil tiene una influencia determinante sobre esta problemática. Uno de esos entornos es el escolar. Si en el colegio o escuela no se tiene claridad sobre los ambientes alimenticios sanos, eso sin duda puede afectar al menor. Si la tienda escolar ofrece con la aquiescencia de las directivas de la institución educativa, comidas no nutritivas y bebidas azucaradas, eso será lo que predomine en la dieta escolar del menor y fuera de la escuela, buscará continuar con esta práctica alimenticia. Estos hábitos podrán trasladarse al entorno familiar. La publicidad desempeña un papel clave en este círculo que se puede romper si el menor está rodeado de ambientes nutricionales más sanos.

En ese contexto, el proyecto analiza si las instituciones educativas departamentales de Colombia han adoptado las políticas públicas en materia de seguridad alimentaria en las tiendas escolares. La investigación se diseñó con un enfoque cuantitativo y cualitativo, ya que con la ayuda de los datos estadísticos que arrojaron 
las respuestas de los derechos de petición, se elaboró un análisis cualitativo del contenido de las políticas públicas de los departamentos, municipios y colegios.

De acuerdo con el cronograma fijado, se enviaron derechos de petición a todos los departamentos $^{2}$ de Colombia y sus capitales, con el fin de verificar la existencia de las citadas políticas públicas. Igualmente, se remitieron derechos de petición a los colegios estatales con mayor cobertura y cantidad de estudiantes de cada departamento. Después de un arduo proceso de gestión ante estas entidades, las respuestas fueron las siguientes: de los 32 departamentos, 24 respondieron el derecho de petición; de las 32 capitales de departamento, 24 respuestas de petición se recibieron; y de los 121 colegios escogidos, 40 respondieron la solicitud. Las cifras recibidas son significativas y con ellas se puede establecer y analizar el cumplimiento de las políticas alimentarias en cada uno de los establecimientos seleccionados.

En la revisión de los derechos de petición dirigidos a las instituciones educativas, se insistió con llamadas telefónicas para solicitar respuesta en el menor tiempo posible. También se comprobó que algunas de las direcciones de colegios que aparecen registradas en la página oficial del Ministerio de Educación no existen, pues el servicio de correos devolvió la correspondencia por dirección inexistente.

El presente artículo consta de dos partes: en la primera se realiza una breve descripción de la exposición de motivos de la ley 1355/2009, "por medio de la cual se define la obesidad y las enfermedades crónicas no transmisibles asociadas a esta como una prioridad de salud pública y se adoptan medidas para su control, atención y prevención", exponiendo el contenido obligacional que surgen a las instituciones educativas; en la segunda se hace la presenta-

2 Colombia tiene 32 departamentos y cada uno de ellos cuenta con una capital que es el centro administrativo y político de la región. ción de los resultados finales de los derechos de petición respondidos por los departamentos, municipios y colegios.

Se precisa que la presente investigación se hizo con base en la información descrita en la Ensin del 2005, sin que hasta la fecha haya una encuesta más reciente. En averiguaciones telefónicas hechas al Ministerio de Salud y Protección Social y al Departamento Administrativo Nacional de Estadística (Dane) se nos informó que para este año se planea dejar listos los instrumentos para aplicar la nueva encuesta que se publicaría en 2017.

\section{A. Ley 1355/2009: la obesidad como una enfermedad crónica de salud pública}

Consultados los archivos del Congreso de la República de Colombia, la ley 1355/2009 tuvo como finalidad declarar la obesidad y las enfermedades crónicas (en adelante EC) asociadas a la misma como problema de salud pública, priorizando la coordinación de acciones de promoción, regulación, monitoreo y vigilancia necesarias para reducir su prevalencia y generar entornos saludables que fomenten su prevención y control mediante la coordinación de acciones dirigidas a implementar la actividad física y la alimentación saludable en los estilos de vida de la población colombiana. Dicha ley determinó obligaciones a las instituciones educativas. Esto en tanto que investigaciones relacionadas con este tema demuestran que:

Las intervenciones dirigidas a mejorar los equipamientos recreativos escolares $e$ incrementar la calidad y el tiempo dedicado a las clases de educación física, han mostrado ser muy efectivas para incrementar los niveles de actividad física, no solo en países desarrollados sino, además, en la población infantil de América Latina. Adicionalmente, algunos estudios han encontrado un efecto marginal, pero significativo, de las clases de educación física en el índice de masa corporal de los niños, que a nivel poblacional podría 
tener un efecto apreciable (Gómez, Ibarra y Lucumí, 2012, p. 12).

La ley 1355/2009 tuvo su justificación en los altos niveles de obesidad que se presentan en la actualidad en Colombia. Según la Organización Mundial de la Salud las EC ocasionan el 60 \% de las muertes en el mundo y el $80 \%$ de las mismas ocurren en países no desarrollados. Contrario a lo que por lo común se asume, este tipo de padecimientos causa un mayor número de muertes prematuras y costos sociales y económicos con respecto a los países ricos. Se estima por ejemplo, que en algunas naciones no desarrolladas como Sudáfrica, el $40 \%$ de las muertes en personas entre los 35 y 60 años es debido a enfermedades cardiovasculares, contrastando con el 10 \% en Estados Unidos en el mismo grupo etario.

Los costos sociales y económicos de las EC tienen efectos especialmente nefastos en países no desarrollados. Así, la pérdida de empleo del proveedor económico de la familia por muerte o secuela de una EC, tiene un efecto negativo en sociedades con economías débiles y deficientes sistemas de seguridad social, generando un incremento en la pobreza y un deterioro en las perspectivas económicas de los países.

Para enfrentar el desafío que representan condiciones crónicas como la obesidad existen estrategias efectivas de salud pública que se orientan sobre todo a la promoción de la actividad física y a una alimentación saludable, lo cual a su vez redundaría en la prevención de otras condiciones crónicas asociadas al sobrepeso y la obesidad.

En Colombia las EC como los padecimientos cardiovasculares y el cáncer son la primera causa de muerte. De acuerdo con el registro de defunciones del Dane del 2005 la tasa de muertes por enfermedad isquémica del corazón en personas de 45 y más años fue de 265,9 por 100000 habitantes, mientras para las enfermedades cerebrovasculares y diabetes fue de 136,4 y 73,0, respectivamente. Estas tasas contrastan con las de todas las causas externas y enfermedades transmisibles en todos los grupos de edad que fueron 80,6 y 33,1 por 100000 habitantes respectivamente.

En cuanto a la obesidad, según la Ensin (2005) el $46 \%$ de los adultos entre los 18 y 64 años padece sobrepeso y obesidad; siendo las prevalencias desagregadas para estos dos problemas del $13,7 \%$ y $32,3 \%$, respectivamente. Los territorios con mayor problema son: Cali $(50,9$ $\%)$, Boyacá, Cundinamarca y Meta $(50,6 \%)$ y Valle sin Cali y sin litoral (50 \%). Por el contrario, los lugares con menores prevalencias son: Atlántico sin Barranquilla, San Andrés y Bolívar norte (37,8 \%), Bolívar sur, Córdoba y Sucre (38,9 \%) y Medellín (40,9\%).

La obesidad abdominal, que está vinculada con riesgo metabólico, reporta una prevalencia en este mismo estudio de 50,4\%. En población joven de 13 a 17 años la Ensin (2005) encontró que $26 \%$ cumple las recomendaciones mínimas definidas para este grupo etario (al menos sesenta minutos de actividades de intensidad moderada o vigorosa por al menos cinco días a la semana).

Este mismo estudio determinó el tiempo dedicado a ver televisión o a jugar con videojuegos en los niños de cinco a doce años, indicador que se conecta con un patrón de vida sedentario y con un consumo mayor de alimentos de alta densidad energética. De acuerdo con esta encuesta, 56,3\% de los niños colombianos ven dos o más horas diarias de televisión, siendo las ciudades de Medellín, Bogotá y Cali las que evidencian más altas prevalencias $(71,9 \%, 70,5$ $\%$ y 70,1\% respectivamente). Las regiones de Bolívar sur, Córdoba y Sucre; Cauca y Nariño sin litoral y litoral Pacífico, presentaron las prevalencias más bajas (39,9\%, 40,9\% y 50,7 \%, respectivamente).

En la subpoblación de niños de cinco a doce años, un análisis secundario hecho con datos de la Ensin 2005 reportó una prevalencia nacional de sobrepeso u obesidad de $11,1 \%$ en aquellos que ven más de dos horas diarias de televisión. 
En cuanto al consumo de frutas y verduras, si bien la Ensin 2005 no permitió establecer cómo se comporta la población colombiana con respecto a la ingesta de las cinco porciones de estos alimentos (400 gramos día), según lo recomendado internacionalmente, sí se pudo determinar que $27,9 \%$ de las personas no incluyó ninguna verdura en su alimentación diaria y 35,3\% no incluyó alguna fruta. Lo anterior puede estar correlacionado con el déficit encontrado en el nivel de vitamina A (32\%) y C $(22,6 \%)$, que ejercen un efecto protector contra las enfermedades cardiovasculares y algunos tipos de cáncer.

Frente al ingreso energético en la Ensin 2005 se reportó que 13,7 \% (IC: 10,8-16,6) de los adolescentes de 14 a 18 años presentó valores excesivos de este indicador (> $110 \%$ ). Un porcentaje importante tenía exceso en el consumo de carbohidratos y grasas saturadas. De igual forma, el porcentaje de adolescentes que dijo haber consumido alguna fruta y verdura en el día fue más bajo que en la población adulta, contrario a lo que ocurrió en el consumo de comidas rápidas.

En ese escenario, la ley 1355/2009 declaró la obesidad como una EC de salud pública, por lo que fijó distintas estrategias para promover una alimentación sana y balanceada, entre ellas, señaló que los establecimientos educativos públicos y privados deben garantizar la disponibilidad de frutas y verduras a sus estudiantes, y que además deben adoptar un programa de educación alimentaria, de conformidad con los lineamientos que desarrolle el actual Ministerio de Salud y Protección Social y el Instituto Colombiano de Bienestar Familiar (ICBF), quienes tendrán en cuenta las características culturales de las distintas regiones de Colombia (art. 4 ley 1355/2009).

En el desarrollo y ejecución de esta ley, la capital del Valle del Cauca se convirtió en un modelo para América Latina, al constituir las "tiendas escolares saludables", en donde se brinda una alimentación balanceada y saludable a los niños de la ciudad. En dicho proyecto se vinculó el sector privado y los resultados han abarcado "aspectos tan importantes como el fomento de la alimentación sana, donde demuestran haber alcanzado metas que transformaron los hábitos de oferta y consumo de alimentos dentro de los planteles con las tiendas escolares" (Secretaría de Educación de Cali, 17 de agosto de 2011).

\section{B. Análisis de los resultados obtenidos en departamentos, municipios y colegios}

\section{Las políticas alimentarias desarro- lladas por los departamentos}

El consolidado de la información indica que el 25 $\%$ de los departamentos no contestó los derechos de petición; el $50 \%$ de los departamentos respondió señalando que no tiene políticas públicas, planes, programas o actividades asociadas con la alimentación escolar; y el $25 \%$ de los departamentos restantes, manifiesta que a pesar de no tener una política pública específica, sí desarrolla actividades o ha implementado estrategias para promover los hábitos de alimentación saludable en su territorio (véase gráfica 1).

\section{Gráfica 1. Consolidado de respuestas de departamentos}

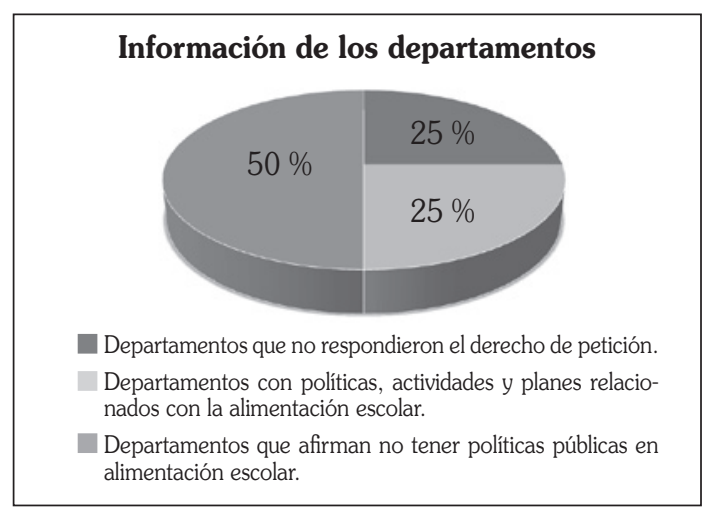

Fuente: elaboración propia.

De las diferentes respuestas sobre las políticas de seguridad alimentaria en colegios públicos se encontró que de los 24 departamentos que 
respondieron, solo ocho cuentan con políticas en seguridad alimentaria (véase tabla 1): Huila, Vaupés, Caquetá, Caldas, Nariño, Quindío, Norte de Santander y San Andrés y Providencia, quienes han regulado de manera congruente y coherente su política con la ley 1355/2009, pues su principal objetivo es prevenir la obesidad en niños, jóvenes y adolescentes.

Tabla 1. Derechos de petición a departamentos

\begin{tabular}{lc}
\hline Derechos de petición sin respuesta & 8 \\
\hline Derechos de petición con respuesta & 24 \\
\hline Total & $\mathbf{3 2}$ \\
\hline
\end{tabular}

Fuente: elaboración propia.

A continuación se expone en detalle las políticas públicas establecidas en los departamentos:

- Quindío, a través de la ordenanza 023/2014 expone paso por paso, la forma en la que se desarrolla en los colegios los programas de alimentación balanceada escolar, que es complementada con charlas didácticas en donde enseñan a los niños y jóvenes la importancia de comer saludable, no solo por gusto, si no por salud, al igual que con distintas estrategias didácticas ayudan a adquirir dichos hábitos.

- La Asamblea Departamental de San Andrés y Providencia expidió la ordenanza 012 de 29 de julio de 2014, que dispone medidas para combatir la obesidad, el sedentarismo y promueve la actividad física, y enfatiza en: (i) el establecimiento de estrategias y medidas especiales de acompañamiento y asesoría a las personas con sobrepeso, obesidad y sedentarismo, así como la promoción de políticas por estilos de vida saludables encabezados por las secretarías de Salud, Educación y Deporte; (ii) la implementación y desarrollo de estrategias para crear hábitos de actividad física y nutricional en los estudiantes, para tales efectos, realizan eventos como la "Semana de hábitos saludables" y la "Celebración del día de la obesidad", así mismo, hacen un registro individual de los estudiantes, a quienes les realizan dos veces al año exámenes de visión, audición, peso y estatura, y registro de niños con sobrepeso y obesidad; y (iii) la imposición a las directivas de las instituciones educativas de ejecutar un estudio de las tiendas, quioscos y restaurantes escolares sobre el valor nutricional de los productos que consumen los estudiantes, y establecer cartilla de alimentos y bebidas saludables en donde se indique los productos que podrán distribuir los restaurantes escolares.

- Caldas, que mediante circular 039 del 23 de marzo de 2016 expedida por la Secretaría de Educación, busca implementar acciones en las instituciones educativas que promuevan hábitos saludables dentro de las tiendas escolares, articulados con el proyecto educativo institucional (PEI).

- Caquetá, a través de la Secretaría de Salud Departamental ha desarrollado actividades enfocadas en la educación y comunicación para la promoción de ambientes saludables mediante la entrega y socialización de cartillas a 380 docentes y 4732 cartillas para colorear a estudiantes denominadas: Cartilla Caquetá un entorno saludable. Estas estrategias estás enmarcadas en lo prescrito en el artículo 13 de la ley 1355/2009.

- Huila, con la ordenanza 035/2010 que adoptó la política pública de seguridad alimentaria y nutricional en el departamento y conformó el Comité de Seguridad Alimentaria y Nutricional, se fijó la estrategia "Escuela saludable" articulando los siguientes componentes: (i) alimentación y restaurante escolar; (ii) inclusión en el PEI tanto del sector público como privado del departamento, del desarrollo de la temática de seguridad alimentaria y nutricional, con el propósito de que se integre en los contenidos de ciencias naturales y educación ambiental, en forma transversal; y los contenidos de educación que se adopten, deben seguir los 
lineamientos y guías que realice el Ministerio de Salud y Protección Social y el ICBF; (iii) promoción de las tiendas saludables en donde se ofrezcan frutas y verduras; (iv) promoción de actividad física de manera regular para prevenir el sedentarismo; (v) implementación de vigilancia nutricional, y (vi) en las sedes educativas del área rural, se promueve el fortalecimiento de programas de huertas escolares y de la cadena alimentaria.

- Nariño, mediante la ordenanza 026/2011 adoptó el "Plan Decenal de Soberanía y Seguridad Alimentaria y Nutricional de Nariño 2010-2020" que procura garantizar el derecho a la alimentación sana y la reducción y prevención de la malnutrición. De igual manera, el Programa de Gobierno del Departamento 2016-2019 "Somos Nariño" busca la cobertura y permanencia de la alimentación escolar, así como promover un estilo de vida saludable, principalmente la alimentación sana.

En dicho documento, se trazan estrategias de cooperación entre el Instituto Departamental de Salud de Nariño, el ICBF y la Secretaría de Educación departamental para implementar el proyecto de tiendas escolares saludables en municipios no certificados; de igual forma, se establecen directrices a la Secretaría Departamental de Educación de Nariño para la oferta de alimentos en las tiendas escolares, dicha secretaría, debe implementar estrategias educativas transversales que desarrollen hábitos saludables a través del PEI, la ejecución de estrategias educativas por parte de los establecimientos educativos en torno al programa de alimentación escolar (PAE) y a los proyectos ambientales escolares (PRAE), procurar por una alimentación equilibrada, suficiente, inocua, variada y adecuada en las tiendas escolares; en ese sentido, determina que el $50 \%$ de la oferta total de las tiendas escolares deberá ser alimentos saludables, el otro $50 \%$ alimentos ricos en grasa y azúcares; así mismo, las tiendas escolares deberán cumplir las disposiciones de la ley 1355/2009. Finalmente, se crea el Comité de Entornos Saludables del municipio, que tendrá la obligación de realizar la supervisión y seguimiento a lo instituido en esta materia en el programa de gobierno.

- Vaupés, a través de la ordenanza 002/2011 expidió el Plan Departamental de Seguridad Alimentaria y Nutricional, de acuerdo con el Plan Nacional de Desarrollo y los objetivos del Conpes, aunque este plan se encamina a la seguridad alimentaria, lo hace de manera general, es decir, no crea obligaciones específicas a las instituciones educativas sino a toda la población del departamento con especial enfoque en niños y niñas. El Plan de Seguridad Alimentaria se concentra en determinar si las fuentes de agua, cosechas y demás son aptas para el consumo de sus habitantes, lo cual hace que dicho plan se desvíe de los objetivos del proyecto de investigación al no abarcar las tiendas escolares ni las instituciones educativas, pese a tener políticas, directrices y programas propios para el bienestar y la seguridad alimentaria de los vaupenses.

- Norte de Santander, no tiene política pública específica, sin embargo, cuenta con la cátedra de salud pública, desarrollando módulos que incluyen temas de nutrición, quedando la implementación al arbitrio de cada rector.

Tabla 2. Consolidado departamentos con política alimentaria

\begin{tabular}{lc}
\hline Departamentos con política alimentaria & 8 \\
\hline Departamentos sin política alimentaria & 16 \\
\hline Total & $\mathbf{2 4}$ \\
\hline
\end{tabular}

Fuente: elaboración propia.

De lo expuesto, se logró determinar que de los ocho departamentos que tienen política alimentaria, cinco han desarrollado su política con base en el PAE y los tres departamentos restantes: 
Nariño, Caquetá y San Andrés y Providencia, han diseñado políticas de alimentación basadas en la ley 1355/2009.

\section{Análisis de las respuestas de los municipios}

Según el diseño del proyecto, se dirigieron derechos de petición a todas las capitales de los 32 departamentos de Colombia con el fin de conocer si los gobiernos municipales habían trazado políticas públicas en materia de alimentación escolar.

Las respuestas de los derechos de petición se pueden reunir en tres grupos: (i) los municipios que no contestaron el derecho de petición (44 $\%$ ); (ii) los municipios que respondieron afirmando que no tienen política pública alimentaria $(25 \%)$ y (iii) los municipios que sí tienen política pública en esta materia y que además desarrollan actividades, planes y programas de sensibilización relacionadas con la alimentación (31\%) (véase gráfica 2).

Gráfica 2. Consolidado de respuestas de los municipios

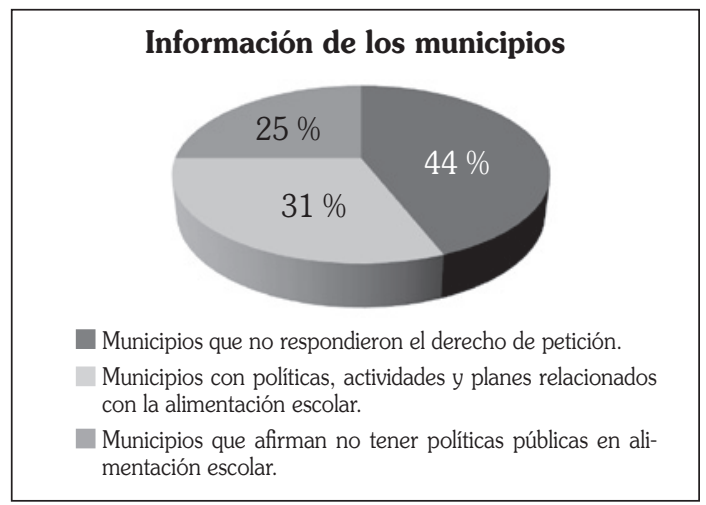

Fuente: elaboración propia.

En el primer grupo se encuentran ocho municipios que no contestaron el derecho de petición, ellos son: Medellín, Quibdó, Florencia, Cartagena, Neiva, Montería, Cúcuta e Ibagué.

En el segundo grupo están catorce municipios que contestaron el derecho de petición informando que no tienen política pública alimentaria, entre ellos están: Leticia (Amazonas), Puerto Carreño (Vichada), Sincelejo (Sucre), Mocoa (Putumayo), Valledupar (Cesar), Riohacha (La Guajira), Inírida (Guainía), San José del Guaviare (Guaviare), Mitú (Vaupés), Pereira (Risaralda), Providencia y Santa Catalina (San Andrés), Tunja (Boyacá), Armenia (Quindío) y Manizales (Caldas) quienes informaron que esta actividad la llevan a cabo de manera autónoma los rectores de cada institución educativa. En este grupo se destaca la respuesta de los siguientes municipios:

- Mocoa (Putumayo). A pesar de que contestó que no tenía una política pública alimentaria, anotó que la administración municipal suscribió el contrato de cooperación 057 del 15 de abril de 2016, para ejecutar e implementar el programa de alimentación escolar a través del cual se brinda un complemento alimentario a los niños, niñas y adolescentes de la matrícula oficial del municipio, de conformidad con los lineamientos técnico-administrativos del programa de alimentación escolar del Ministerio de Educación Nacional. El proyecto permitió ampliar cobertura mediante la estrategia alimentación escolar; donde el objetivo principal fue contribuir a mejorar el desempeño académico y la asistencia regular, así como también promover la formación de hábitos alimentarios saludables.

- Sincelejo (Sucre). Indicó que el 23 de enero de 2014 se ofició el convenio 001/2014 de asociación, cooperación, cofinanciación y aportes celebrado entre Sincelejo y la Fundación Prosperar Colombia, que tenía como objetivo principal realizar el suministro de complementación alimentaria para la promoción de un estilo de vida saludable que contribuyera con la permanencia de los estudiantes.

- Puerto Carreño (Vichada). Señaló que en el Plan Territorial de Salud Pública en la dimensión de seguridad alimentaria y nutricional se han previsto acciones de consumo 
de alimentos saludables en las instituciones educativas.

- Inírida (Guainía). Respondió que no se han aprobado políticas, sin embargo, desde el Programa de Salud Pública se desarrollan actividades para evitar la obesidad infantil, existe comité técnico del Plan de Seguridad Alimentaria y Nutricional Departamental y Municipal creado mediante decreto 0482/30 de noviembre de 2009, se enmarca en la Política Nacional de Seguridad Alimentaria y Nutricional (documento Conpes social 113/2008).

En el tercer grupo están los diez municipios que respondieron confirmando la existencia de políticas públicas en esta materia, que se detallan a continuación:

- Arauca. La alcaldía afirmó que a través de la dimensión seguridad alimentaria y nutricional, ejecutó proyectos de salud pública, campañas de promoción de la alimentación saludable en instituciones educativas públicas urbanas del municipio, en donde se incluyó: (i) el seguimiento a la alimentación saludable que consumen los estudiantes en las instituciones educativas públicas de Arauca; (ii) visitas a cada una de las cafeterías con el objeto de educar y verificar el cumplimiento de la ley 1355/2009; y (iii) visitas a las instituciones educativas para sensibilización y aplicación de una encuesta a sus docentes y rectores, con el propósito de conocer cómo perciben la cafetería de su institución y qué compromisos desarrollarían para mejorarla. Finalmente, indicaron que el municipio cuenta con el comité de seguridad alimentaria y nutricional que actúa como órgano asesor en esta materia y el sector educación es uno de los actores de dicho comité.

- Barranquilla. Informó que se aprobó el acuerdo 0025/2013 por medio del cual se estableció como objetivo evitar la obesidad infantil en colegios públicos y privados, además procura por mantener el peso ideal de niños y adolescentes estipulando los programas que se implementarán en las instituciones, de igual manera los métodos para dicho propósito tales como publicidad, capacitaciones, entre otros.

- Yopal. La Secretaría de Educación y Cultura del municipio expidió la resolución $1250 \mathrm{del}$ 20 de noviembre de 2014 que promueve la oferta y disponibilidad de alimentos saludables y de alto valor nutricional en las tiendas escolares de las instituciones educativas públicas y privadas de Yopal, haciendo énfasis en: (i) la distribución y venta de alimentos saludables de alto valor nutricional y la restricción gradual de alimentos de alta densidad calórica y bajo valor nutricional; y (ii) la implementación de las tiendas escolares saludables, en donde los estudiantes deben tener disponibilidad y acceso a alimentos saludables e inocuos en un plazo máximo de seis meses, para tales efectos, la Secretaría de Educación y Cultura municipal hará el seguimiento y evaluación.

Las medidas adoptadas por el municipio se realizaron considerando el Comité de Seguridad Alimentaria y Nutricional, que se creó mediante decreto 120/2010 de la alcaldía; el Plan de Desarrollo "Yopal con Sentido Social 2012-2015" que contempla acciones en seguridad alimentaria y nutricional en los ejes social, económico y productivo, territorial y de gestión y gobierno; y el Plan Municipal de Seguridad Alimentaria y Nutricional "Con Sentido Nutritivo 2011-2020" aprobado mediante acuerdo municipal 013/2013 que tiene como objetivo mejorar la calidad de vida de los habitantes.

- Popayán. Afirmó en su respuesta que con el acuerdo 24 de diciembre de 2010 se reglamentó en ocho artículos los organismos encargados y la forma en que se evitarían alimentos no saludables en las tiendas escolares.

- Bogotá. Respondió sosteniendo que la aprobación del acuerdo distrital 086/2003 crea 
el sistema distrital de vigilancia alimentaria para niños y niñas preescolares y escolares.

- Santa Marta. Expresó que desde la Secretaría Seccional de Salud se difunde la aplicación de la ley 1355/2009 ejecutando actividades como: (i) capacitación para la promoción de modos, condiciones y estilos de vida saludables con énfasis en la niñez y la adolescencia, y (ii) procesos de formulación de política pública que promuevan modos, condiciones y estilos de vida saludables por parte de la administración departamental.

- Villavicencio. Expresó que dentro del Plan de Desarrollo "Unidos Podemos", se vinculó la implementación de las tiendas escolares saludables: (i) gestionar en las instituciones educativas proyectos de estilos de vida saludable, y (ii) gestionar en las mismas la creación de tiendas escolares saludables.

- Pasto. Argumentó que mediante acuerdo 036 de 31 de octubre de 2014, se aprobó el Plan de Seguridad Alimentaria y Nutricional, Pasto Asegurando su Alimentación y Nutrición, consolidando la adecuada oferta y mejora de los hábitos alimentarios. Igualmente, expidió la resolución 0637 de 8 de octubre de 2015, que fija las directrices para el funcionamiento y oferta de alimentos de las tiendas escolares de los establecimientos educativos públicos y privados de Pasto. Entre otras obligaciones, ordenó que las tiendas escolares deberán propiciar una alimentación saludable e inocua, los alimentos expendidos bajo marca deberán contar con registro sanitario.

Así mismo, determinó que las secretarías de Educación y Agricultura, las plazas de mercado y las instituciones educativas darán a conocer un listado de alimentos que sean fuente de proteína, grasas saludables, carbohidratos complejos, fibra, vitaminas y minerales. Además, prevé que las instituciones educativas con sus comités, coordinarán acciones para concientizar e influir en los estudiantes la elección de alimentos y estilo de vida saludables con ayuda de propaganda alusiva a ello. También realizarán actividades relacionadas con el Día de la Obesidad y el Día Mundial de la Alimentación. Vincula a la Secretaría de Educación para verificar el cumplimiento de las estipulaciones de la resolución en los contratos celebrados entre las instituciones y las tiendas escolares.

- Bucaramanga. Con el acuerdo municipal 020 de 27 de julio de 2012 se determina la política pública para el control y prevención de la obesidad. Ordena a la Secretaría de Salud y Ambiente en conjunto con la Secretaría de Educación, implementar conforme con los programas establecidos en el plan de desarrollo, estrategias de promoción y sensibilización de estilos de vida saludable en los establecimientos educativos oficiales y privados del municipio.

- Cali. Confirmó la aprobación de una política pública en la materia con fundamento en la ley 1355/2009, consolidada en el acuerdo 0278/2009 que exige el aumento de la oferta de alimentos saludables, de alto valor nutricional e inocuos en las tiendas, quioscos, máquinas expendedoras y restaurantes escolares en todas las instituciones educativas públicas y privadas de la ciudad.

Cada municipio cuenta con su repectivo acuerdo del Concejo Municipal y plantea claramente la necesidad de la aplicación de la ley 1355/2009 y su interés por prevenir y bajar los índices de obesidad en menores de edad y poder tener una alimentación escolar balanceada.

No obstante, de los municipios capitales de departamento, algunos nos informan que pese a no tener estrictamente una política pública con relación a la ley 1355/2009, desarrollan actividades de sensibilización u otros distintos programas dirigidos a la alimentación en los colegios. 


\section{Análisis de las respuestas de las instituciones educativas}

En total se seleccionaron 121 instituciones educativas nacionales, esta se hizo teniendo en cuenta la cantidad de estudiantes del respectivo colegio, lo cual le daba un peso significativo a la selección. La tabla 3 ilustra el número de establecimientos educativos escogidos por departamento.

Tabla 3. Cantidad de colegios seleccionados por departamentos

Departamento Cantidad Departamento Cantidad

\begin{tabular}{llll}
\hline Amazonas & 2 & Guaviare & 4 \\
\hline Antioquia & 6 & Huila & 3 \\
\hline Arauca & 3 & La Guajira & 3 \\
\hline $\begin{array}{l}\text { Archipiélago } \\
\text { de San Andrés, }\end{array}$ & 4 & Magdalena & 4 \\
$\begin{array}{l}\text { Providencia y } \\
\text { Santa Catalina }\end{array}$ & & & \\
\hline
\end{tabular}

\begin{tabular}{llll}
\hline Atlántico & 5 & Meta & 4 \\
\hline Bolivar & 6 & Nariño & 4 \\
\hline Boyacá & 3 & Norte Santander & 3 \\
\hline Caldas & 3 & Putumayo & 3 \\
\hline Caquetá & 2 & Quindío & 5 \\
\hline Casanare & 3 & Risaralda & 6 \\
\hline Cauca & 5 & Santander & 4 \\
\hline Cesar & 4 & Sucre & 4 \\
\hline Chocó & 4 & Tolima & 3 \\
\hline Cundinamarca & 2 & Valle del Cauca & 6 \\
\hline Córdoba & 4 & Vaupés & 4 \\
\hline Guainía & 2 & Vichada & 3 \\
\hline Total & & 121
\end{tabular}

Fuente: elaboración propia.

De las 121 instituciones educativas que se seleccionaron para adelantar esta investigación, se recibieron 40 respuestas, de las cuales tan solo 14 afirman tener una política de alimentación saludable, con base en lo ordenado en la ley $1355 / 2009$, desarrollando actividades que se reflejan ejerciendo un estricto control sobre las tiendas escolares. Por su parte, 26 instituciones educativas respondieron aseverando que no tenían ninguna política pública, plan o acción asociada con dicha normativa (véase gráfica 3). Algunas de las instituciones educativas que han implementado programas, planes o acciones en el marco de la ley 1355/2009 son:

El Colegio de Boyacá, de Tunja, en virtud de la ley $1355 / 2009$ ha adelantado programas que se encaminan hacia la seguridad alimentaria, por ello tiene un estricto control sobre lo que se vende en las tiendas escolares, donde solo se encuentran productos que aportan nutrientes a la salud de sus estudiantes, así mismo ha intensificado las clases de educación física para así cumplir con un circuito tanto en alimentación como en salud física.

En Florencia, la institución educativa San Francisco de Asís, con fundamento en la ley 1355/2009 creó medidas de aprovechamiento del tiempo libre con actividades de carácter lúdico. En el consejo estudiantil se abordó la problemática de la escasez de alimentos saludables y se decidió propiciar charlas educativas con respecto al tema.

El colegio Normal Superior de Montería respondió indicando que a la luz del artículo 11 de la ley $1355 / 2009^{3}$ ofrece alimentos y bebidas que cumplen con los nutrientes necesarios para el desarrollo y la salud de sus estudiantes.

En el mismo sentido, la institución educativa Las Palmas de Villavicencio señaló que de acuerdo con la ley 1355/2009, en particular el artículo 11, brinda alimentos y bebidas a través del refrigerio escolar, suministrado según

3 La citada norma prescribe que: "Las instituciones educativas públicas y privadas que suministren el servicio de alimentación de manera directa o a través de terceros, deberán ofrecer una diversidad de alimentos que cubran las necesidades nutricionales de su comunidad, siguiendo, entre otras referencias, las guías alimentarias del Ministerio de la Protección Social y del ICBF, velando por la calidad de los alimentos que se ofrecen y de conformidad con los lineamientos establecidos por el Ministerio de la Protección Social a que se refiere el artículo $8^{\circ}$ de la presente ley". 
las tablas nutricionales del ICBF y por medio de terceros en la tienda escolar. No existe una política, plan o norma escrita que se conecte con la alimentación que se vende en la tienda escolar que indique el tipo de alimentos que se puede vender o no.

La institución educativa Ciudad de Pasto de San Juan de Pasto posee un acuerdo institucional sobre el manejo y funcionamiento de la cafetería escolar, que deberá satisfacer las normas de consumo saludable, aseo y seguridad. Además, dispone qué clase de productos se comercializarán, propendiendo por alimentos saludables.

Por su parte el colegio distrital Olaya de Barranquilla manifestó que en el marco de la ley 1355/2009 adoptó un plan de medidas tendientes a mejorar la salud alimenticia de sus estudiantes. Estas medidas están planificadas en común acuerdo con el administrador de la tienda escolar y también se están evaluando recomendaciones del programa PAE.

La institución educativa Manuela Beltrán de San José del Guaviare argumentó que para cumplir la ley 1355/2009 ofrece capacitaciones a los encargados de las tiendas escolares, cursos de manipulación y programas de día saludable.

En otro grupo podemos incluir a las instituciones educativas que no tienen políticas públicas y no desarrollan actividades en cumplimiento de la ley 1355/2009, ellas son:

La institución educativa Kennedy de Bogotá expidió la resolución 16240/2002 que indica qué es una regulación a la seguridad alimentaria en comedores escolares; sin embargo, la norma reglamenta la oferta de servicios alimenticios que se ofrecen en dicha institución educativa, en aspectos relacionados con el pliego de condiciones, los horarios de atención a estudiantes, el canon de arrendamiento y la responsabilidad en equipos de cocción de alimentos. Aclarando que la citada norma no establece el tipo de alimentos que se brindarán a los estudiantes ni mucho menos contenido, peso y vitaminas.
El colegio Camilo Torres de Cartagena arguye que a los estudiantes se les suministra alimentación de buena calidad pero es un poco incoherente cuando mencionan que se venderán gaseosas en envases y bolsas de buena calidad, esto debido a que dichas bebidas no contribuyen a la buena alimentación de los alumnos, así mismo en acta 28 de 26 de mayo 2016 se deja presente solicitud para realizar un estudio sobre la calidad de alimentos que se venden en la tienda escolar.

Por su parte, el colegio Bertha Gedeón de Baladí de Cartagena manifestó que es el gobierno el responsable de asignar tales políticas a las instituciones educativas públicas y que ellos cumplen con incentivar a los estudiantes en sus clases de ciencias naturales sobre la alimentación mas no tienen políticas sobre seguridad alimentaria.

En Popayán, la institución educativa San Agustín declaró no tener políticas propias sobre seguridad alimentaria; aunque en las cláusulas 2 y 3 del contrato suscrito con la entidad encargada de suministrar los alimentos a la tienda escolar se especifica que se deben vender alimentos saludables y no tóxicos a los estudiantes.

El Instituto Técnico Nacional de Comercio de Barranquilla indicó que a pesar de que no cuenta con políticas de seguridad alimentaria, sí ha intensificado la venta de frutas y prohíbe una vez a la semana la venta de gaseosas.

La Escuela Normal Superior de Manizales apuntó que aunque no cuenta con una política de seguridad alimentaria, tiene la supervisión del ICBF, entidad responsable de proveer los alimentos saludables a los estudiantes como frutas, cereales, leche y agua, expresando que a los estudiantes de primaria no les proporcionan bebidas artificiales, tales como gaseosas.

En Arauca, la institución educativa Santa Teresita no tiene políticas en seguridad alimentaria pero sí ha realizado encuestas a sus cafeterías y ha hecho una socialización de los productos que se deberían ofrecer a los estudiantes. 
La institución educativa Rufino Centro de Armenia cuenta con el restaurante escolar implementado por el Gobierno nacional, por lo cual la tienda escolar prácticamente se reduce a la venta de productos que consumen los docentes del plantel; pero no se tiene establecida una reglamentación sobre la norma en cita.

El colegio María Doraliza López de Mejía de Riohacha viene adelantando una encuesta con los estudiantes y padres de familia para hacer un listado de los alimentos que deberían vender en la tienda escolar, para dar cumplimiento a la ley 1355/2009. En el mismo sentido, se encuentra la institución educativa El Diamante de Medellín, que apuntó que está en proceso de consolidación de sus políticas sobre seguridad alimentaria.

La Escuela Normal Superior "El Jardín" de Pereira adjuntó una circular de la Secretaría de Salud que dice cumplir a cabalidad, en la cual se determina cómo será el manejo de productos comestibles, pero no aclara qué productos son los comercializados, simplemente se enuncian los términos de productos de nutrición, saludables y de fácil acceso económico.

En general, es evidente cómo el campo de acción y regulación en los colegios sobre políticas alimentarias está abandonado o dejado de lado, ya que en las respuestas de los colegios a los derechos de petición, ni la mitad de ellas son positivas $y$ en muchas declaran que es competencia del Estado y no del colegio regular en esta materia.

De las instituciones educativas que afirman tener políticas públicas en la materia, al analizarlas no ostentan tal calidad. En efecto, el diseño de una política pública requiere de conocimientos en economía, ciencia política, estadística, administración pública, entre otros. Por lo tanto, se necesita de un equipo de trabajo multidisciplinario y de una información precisa que en muchos casos las instituciones gubernamentales no disponen. El diagnóstico y estudio del problema son muy importantes con el fin de determinar cuáles son las soluciones más reales y aplicables y cuál es el presupuesto requerido, el análisis de factibilidad y la toma de la decisión.
Gráfica 3. Respuestas de los colegios

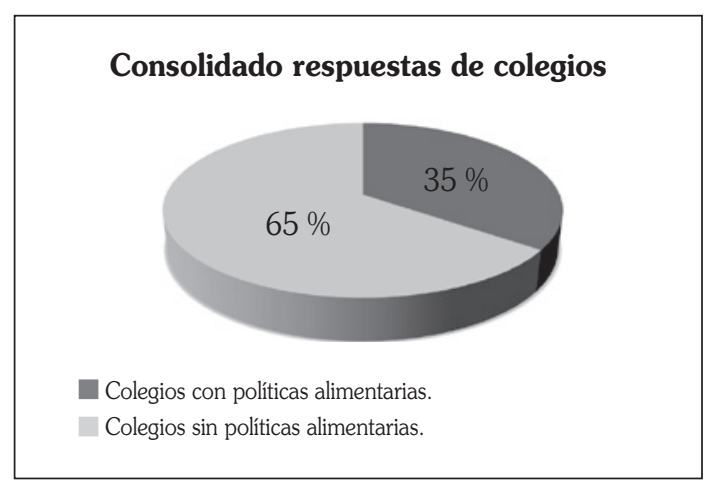

Fuente: elaboración propia.

\section{Conclusiones}

El Ministerio de Educación ha adelantado estrategias de promoción de la ley 1355/2009 en las instituciones educativas, como por ejemplo, cuando declaró la Semana de Hábitos de Vida Saludables (del 22 al 25 de septiembre de 2015), con el fin de

[...] ofrecer a las entidades territoriales orientaciones conceptuales, pedagógicas y operativas para guiar a los establecimientos educativos a la construcción de proyectos pedagógicos transversales, que contribuyan al desarrollo de habilidades y actitudes de los niños y niñas de manera que tomen decisiones pertinentes frente a su salud, su alimentación, su crecimiento, su desarrollo cognitivo y su proyecto de vida, y que aporten a su bienestar individual y al colectivo (Ministerio de Educación, 2015, p. 1).

A pesar de dichas estrategias, de los datos obtenidos se tiene que la mayoría de los municipios, departamentos y colegios estatales a los que se les enviaron derechos de petición, no tiene políticas públicas en materia de alimentación sana en tiendas escolares.

Uno de los pocos departamentos que regula de manera detallada el tema es el Quindío. De acuerdo con la ordenanza 023/2014 se fija paso a paso la forma en la que se debe desarrollar en los co- 
legios dos programas de alimentación balanceada escolar, los cuales deben complementarse con charlas didácticas y técnicas que enseñan a los niños y jóvenes la importancia de comer saludable.

En los documentos que contienen las supuestas políticas públicas, se observa y verifica en la mayoría de los casos que los departamentos y municipios en sus respectivos actos administrativos (ordenanzas, acuerdos o resoluciones), fijan unos parámetros muy generales en los que se tratan de implementar algunas acciones tendientes a hacer frente a una problemática que no está bien delimitada, ni identificada. Por tanto, las medidas tomadas en algunos casos no son las más eficaces.

Por su parte, de los 24 municipios que respondieron el derecho de petición solo diez cuentan con política pública estructurada. Entre estos municipios se destaca Barranquilla que aprobó un acuerdo municipal (el 0025/2013) por medio del que se precisa como objetivo evitar la obesidad infantil en colegios públicos y privados, así como el peso ideal de niños y adolescentes. En 23 artículos, el Consejo Municipal estableció algunas medidas como la eliminación en los planteles educativos de las bebidas azucaradas y grasosas, la implementación de estadísticas en obesidad y sobrepeso, campañas de actividad física, nutrición infantil e involucrar a las familias en estas campañas. Además, se adoptó el 24 de septiembre como el día de la lucha contra la obesidad. Se instituyó una remisión obligatoria de los niños con obesidad a su respectiva empresa promotora de salud para que inicie un seguimiento.

Consideramos que este acuerdo municipal y el de Yopal son los más completos entre los recibidos de las diferentes entidades municipales, pues en la regulación de este último municipio se incita a consumir alimentos saludables y de alto valor nutricional en las tiendas escolares. El documento contiene lo relacionado con la distribución y expendio de alimentos saludables de alto valor nutricional y restringe gradualmente los alimentos de alta densidad calórica y bajo valor nutricional.
Igualmente ordena a las tiendas escolares la venta, disponibilidad y acceso de alimentos saludables e inocuos en un plazo máximo de seis meses.

No menos importante es el caso del municipio de Pasto, que mediante acuerdo 036 de 31 de octubre de 2014 aprobó el Plan de Seguridad Alimentaria y Nutricional que procura el mejoramiento de los hábitos alimentarios de toda la población del municipio. Es decir, no está dirigido de manera exclusiva a los colegios. Es un acuerdo muy general que no va enfocado a la población infantil de las instituciones educativas.

En el caso de los colegios que sí tienen política pública, podemos hacer un análisis de la forma como estos tratan de implementar medidas para hacer frente a la malnutrición infantil. En la respuesta del Colegio de Boyacá, de Tunja (una de las pocas que sí tiene) se verifica que la implementación de la política alimentaria y la prevención de la obesidad infantil se basa en el impulso de hábitos saludables tales como la ingesta de frutas, lácteos, cereales y agua así como la intensificación de clases de educación física y el entrenamiento deportivo en diferentes disciplinas. A pesar de ser la respuesta más completa de las recibidas, no se expone el modo como se impulsan estas medidas ni los resultados de ellas.

De las respuestas allegadas por parte de departamentos, municipios y colegios, se pudo constatar que no existe claridad en cuanto a la forma de establecer una política pública en materia alimentaria. Además, se evidenció que las políticas públicas en esta área son escasas en el país, por lo que el Estado deberá tomar medidas con el fin de que el contenido de la ley $1355 / 2009$ se aplique.

Se indagó por las entidades que no tienen una política pública en la materia y se pudo comprobar de manera informal (telefónicamente) que en la mayoría de los casos es por desconocimiento de la ley, por desconocimiento de la problemática en su respectivo colegio o simplemente por desidia administrativa. Es decir, en algunos casos 
es un asunto de segundo nivel que no atrae la atención de la administración pública.

Lo anterior, permite afirmar de manera categórica que son muy pocos los colegios que se ocupan de este tema de la política en materia alimentaria de los menores de las instituciones educativas. El desconocimiento de la norma, así como el poco interés en el asunto pueden ser principalmente las causas de este vacío.

Consideramos que se deben seguir adelantando estrategias que den a conocer las obligaciones que prescribe la ley 1355/2009, para que las instituciones educativas realicen con los estudiantes de preescolar, primaria y bachillerato actividades lúdicas, deportivas y recreativas para que los niños y jóvenes aprendan sobre los hábitos de vida saludable, puesto que como lo expresa Fajardo (2012) es más fácil enseñar que modificar hábitos, dado que en esto último, se generan mayores costos en las intervenciones y mayores fracasos. Así, con estrategias educativas dirigidas a los niños y adolescentes se les estimulará a tener la salud adecuada y la energía necesaria para realizar las actividades propias de esta etapa de la vida.

\section{Referencias}

Amador, E. M. \& Montealegre, L. P. (2011). Sobrepeso obesidad infantil. Una mirada desde la fisioterapia. Revista Academia Libre, Universidad Libre de Barranquilla, 8(9), pp. 129-134.

Banco Interamericano de Desarrollo. (2012). Nutrición en Colombia II Actualización del estado nutricional con implicaciones de política. Disponible en: https:// publications.iadb.org/bitstream/ handle/11319/5932/28May2013\%20 NT\%20nut.pdf?sequence $=1$

Baile, J. I. (2007). Obesidad infantil ¿qué hacer desde la familia? Madrid: Síntesis.

Chueca, M., Azcona, C. \& Oyarzábal, M. (2002). Obesidad infantil. Anales Sistema Sanitario de Navarra, 25, pp. 127-141.
Colomer, J. (2005). Prevención de la obesidad infantil. Pediatría Atención Primaria, VII(26), abril-junio, pp. 79-99.

Fajardo, E. (2012). Obesidad infantil: otro problema de malnutrición. Revista Med, 20(1), enero-junio, pp. 6-8.

Fajardo, E. \& Ángel, L. A. (2012). Prevalencia de sobrepeso y obesidad, consumo de alimentos y patrón de actividad física en una población de niños escolares de la ciudad de Bogotá. Revista Universidad Militar, 20(1), enero-junio 101-116.

Gómez, L. F., Ibarra, L. \& Lucumí, D. (2012). Alimentación no saludable, inactividad física y obesidad en la población infantil colombiana: un llamado urgente al Estado y la sociedad civil para emprender acciones efectivas. Disponible en: https://www.researchgate.net/publication/262108142_Alimentacion_no_saludable_inactividad_fisica_y_obesidad_en_ la_poblacion_infantil_colombiana_Un_llamado_urgente_al_estado_y_la_sociedad_ civil_para_emprender_acciones_efectivas

Ministerio de Educación Nacional. (2015). Propuesta de actividades en el marco de la Semana de Estilos Saludables. Disponible en: http:// www.mineducacion.gov.co/cvn/1665/ articles-353950_archivo_pdf.pdf

Ministerio de la Protección Social (2010). Encuesta nacional de la situación nutricional en Colombia 2010. Disponible en: https:// www.minsalud.gov.co/sites/rid/Lists/ BibliotecaDigital/RIDE/VS/ED/GCFI/ Base $\% 20$ de $\% 20$ datos $\% 20$ ENSIN $\% 20$ -\%20Protocolo\%20Ensin\%202010.pdf

Muzo, S. (2003). Crecimiento normal y patológico del niño y del adolescente. Revista Chilena de Nutrición, 30, pp. 92-100.

Secretaría de Educación de Cali. (17 de agosto de 2011). Tiendas escolares saludables de Cali, modelo para América Latina. Disponible en: http://www. cali.gov.co/educacion/publicaciones. php?id $=41014 \& d$ Print $=1$ 